\title{
EXISTENCE OF SOLUTIONS FOR AN ELLIPTIC-ALGEBRAIC SYSTEM DESCRIBING HEAT EXPLOSION IN A TWO-PHASE MEDIUM
}

\author{
Cristelle Barillon ${ }^{1}$, Georgy M. Makhviladze ${ }^{2}$ and Vitaly A. Volpert ${ }^{3}$
}

\begin{abstract}
The paper is devoted to analysis of an elliptic-algebraic system of equations describing heat explosion in a two phase medium filling a star-shaped domain. Three types of solutions are found: classical, critical and multivalued. Regularity of solutions is studied as well as their behavior depending on the size of the domain and on the coefficient of heat exchange between the two phases. Critical conditions of existence of solutions are found for arbitrary positive source function.
\end{abstract}

Mathematics Subject Classification. 36J70, 80A20.

Received: July 7, 1998. Revised: October 20, 1999.

\section{INTRODUCTION}

There is a big physical literature devoted to heat explosion in heterogeneous media (see for example [1,2,6, $8,11]$ ). In this paper we study one of the models which describes heat explosion in a non-moving heterogeneous medium consisting of reacting particles surrounded by a gas. Chemical reaction can occur inside the particles or between the particles and the gas, but it does not occur inside the gaseous phase. The particles are sufficiently small and there is no heat conduction in this phase. The gas is heat conducting and there is heat exchange between the two phases. The heterogeneous medium heated due to exothermic chemical reaction loses its energy by heat conduction between the gas and the vessel's cold boundaries which are maintained at the initial (room) temperature. We denote $u_{1}$ and $u_{2}$ the dimensionless temperatures of the particles and the gas respectively, a nonlinear function $F\left(u_{1}\right)$ characterizes the rate of heat production in the particles' phase, the parameter $\alpha$ the rate of heat exchange between two phases. This model is represented in the stationary case by the following elliptic-algebraic system of equations

$$
\begin{aligned}
F\left(u_{1}\right)-\alpha\left(u_{1}-u_{2}\right) & =0, \\
\Delta u_{2}-\alpha\left(u_{2}-u_{1}\right) & =0,
\end{aligned}
$$

which can be understood as equilibrium of heat production and heat loss in each phase. Equations (1.1), (1.2)

Keywords and phrases. Elliptic - algebraic equations, heat explosion, classical and critical solutions, topological degree, continuous branches of solutions, minimax representation.

1 School of Mathematical Sciences, Tel Aviv University, Ramat Aviv, Tel Aviv 69978, Israel.

2 Center for Research in Fire and Explosion Studies, University of Central Lancashire, Preston, PR1 2HE, UK.

3 Analyse Numérique, UMR 5585 CNRS, Université Claude Bernard Lyon I, 69622 Villeurbanne Cedex, France. 
are considered in a bounded domain $\Omega \subset \mathbb{R}^{m}$ with a sufficiently smooth boundary $\partial \Omega$. The boundary condition is

$$
\left.u_{2}\right|_{\partial \Omega}=0 .
$$

The function $F\left(u_{1}\right)$ is supposed to be positive for $u_{1} \geq 0$, the parameter $\alpha$ is also positive.

The basic question in the theory of heat explosion is to find critical conditions of existence of solutions depending on parameters of the problem and, in particular, on the size of the domain. Heat explosion in a two-phase medium is considered in $[6,11]$ in the case where the gas temperature does not depend on the spatial variable. One dimensional spatial case for a particular form of the non-linearity $F(u)=\exp (u)$ is studied numerically in [2]. In this work we consider the multidimensional case for an arbitrary positive function $F(u)$.

We suppose that the domain $\Omega$ is star-shaped, i.e. if 0 is a "star-shaping" point, for any $x_{0} \in \Omega$ the interval $t x_{0}, 0 \leq t \leq 1$ also belongs to $\Omega$. In this case we can introduce a family of domains $\Omega_{\lambda}$ each of them obtained from the domain $\Omega$ by the transformation $y=\lambda x$, where $y \in \Omega_{\lambda}, x \in \Omega$. The parameter $\lambda$ determines the size of the domain, and so $\lambda_{1}>\lambda_{2}$ implies the inclusion $\Omega_{\lambda_{2}} \subset \Omega_{\lambda_{1}}$. Then, considering the problem (1.1),(1.2) in a domain $\Omega_{\lambda}$ of size $\lambda$, the system can be written, via a change of variables, in the domain $\Omega$ as

$$
\begin{aligned}
F\left(u_{1}\right)-\alpha\left(u_{1}-u_{2}\right) & =0 \text { in } \Omega, \\
\Delta u_{2}-\lambda^{2} \alpha\left(u_{2}-u_{1}\right) & =0 \text { in } \Omega, \\
\left.u_{2}\right|_{\partial \Omega} & =0 .
\end{aligned}
$$

A sum of equations (1.3) and (1.4) gives the following equation

$$
\Delta u_{2}+\lambda^{2} F\left(u_{1}\right)=0 .
$$

We can expect from (1.3) that the temperatures $u_{1}$ and $u_{2}$ of the two phases become close to each other as $\alpha$ the coefficient of heat exchange increases. In this case we obtain the following limiting problem

$$
\Delta v+\lambda^{2} F(v)=0,\left.\quad v\right|_{\partial \Omega}=0 .
$$

We show that indeed solutions of the problem (1.3)-(1.5) converge to nondegenerate solutions of (1.7) as $\alpha \rightarrow \infty$ (Sect. 3). Critical conditions of existence and behavior of solutions of the problem (1.7) are well studied (see for example $[4,5,9,14]$ ).

For arbitrary positive $\alpha$ the situation can be more complicated. The system (1.3), (1.4) is a differentialalgebraic system (for the case of ordinary differential and algebraic systems of equations see [10] and references therein). A natural approach to study it is to express one of the variables from the first equation and to substitute it into the second one. It should be noted, however, that there can exist critical points where $F^{\prime}\left(u_{1}\right)=\alpha$. In this case, if we express $u_{2}$ through $u_{1}$ from (1.3) and substitute into (1.4), we obtain a degenerate semilinear elliptic equation, while, if $u_{1}$ is expressed though $u_{2}$, we obtain an equation with a multivalued nonlinearity. Existence of the critical points determines the type of solutions. We show that there are two types of solutions: classical and critical (Sect. 2). A classical solution is a singlevalued vector function with $u_{i}(x) \in C^{(2, \delta)}(\bar{\Omega}), i=1,2$ and $F^{\prime}\left(u_{1}(x)\right)<\alpha$ everywhere inside $\bar{\Omega}$. For a critical solution $F^{\prime}\left(u_{1}(x)\right) \leq \alpha$ and there exists a critical set where $F^{\prime}\left(u_{1}(x)\right)=\alpha$. There are also multivalued solutions that would appear in the case where $F^{\prime}\left(u_{1}(x)\right)$ exceeds $\alpha$. Figure 1 represents a schematic view of those different types of solutions for a one-dimensional domain. The temperature of the particles $u_{1}$ is plotted on the interval: classical solutions are represented by a smooth profile, for critical solutions, the profile looses its regularity at a critical point $x^{*}$ where the particles' temperature reaches the critical value $u_{1}^{*}$ and $F^{\prime}\left(u_{1}^{*}\right)=\alpha$. The third curve on this figure gives an idea of what a multivalued solution looks like.

The contents of the paper are as follows. In Section 2 we study regularity of solutions. We show in particular that the critical solutions are Hölder continuous and that the critical set has zero measure. Section 3 is devoted 


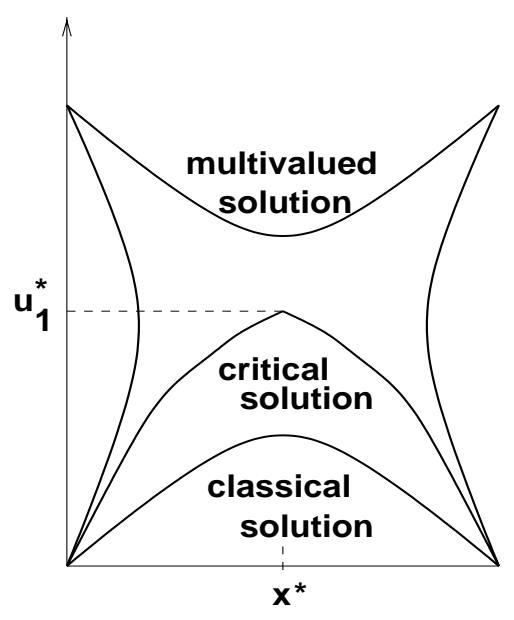

FiguRE 1. 3 types of solutions.

to existence of solutions. As it was already mentioned we analyze the behavior of solutions for large $\alpha$. We prove also existence of continuous branches of solutions depending on the parameter $\lambda$. Each such branch ends with a critical solution which separates classical and multivalued solutions. We show that bounded solutions do not exist if the domain is sufficiently large. In Section 4 we obtain a critical condition for existence of solutions in the form of a minimax representation assuming that $F\left(u_{1}\right)$ is a convex function. It allows to find or to estimate the critical size of the domain above which there is no bounded stationary solution to the system. This absence of bounded stationary solution is interpreted as heat explosion.

\section{Estimation of Classical SOLUTiOnS}

We assume that the function $F(s)$ is positive and continuous with its second derivatives. We recall that $\lambda$ and $\alpha$ are positive parameters. Put $G_{\alpha}(s)=s-\frac{1}{\alpha} F(s)$. Suppose that there exists a positive solution $u_{1}^{0}$ to the equation $G_{\alpha}(s)=0$ such that $G_{\alpha}^{\prime}\left(u_{1}^{0}\right)>0$. If $G_{\alpha}^{\prime}(s)>0$ for all $s \geq u_{1}^{0}$, then $u_{2}(x)$ can be expressed through $u_{1}(x)$ from (1.3) and we obtain the problem:

$$
\begin{gathered}
\operatorname{div}\left(G_{\alpha}^{\prime}\left(u_{1}\right) \nabla u_{1}\right)+\lambda^{2} F\left(u_{1}\right)=0, \\
\left.G_{\alpha}\left(u_{1}\right)\right|_{\delta \Omega}=0
\end{gathered}
$$

equivalent to (1.3)-(1.5). Assume now that there exists $u_{1}^{*}>u_{1}^{0}$ such that $G_{\alpha}^{\prime}\left(u_{1}^{*}\right)=0, G_{\alpha}^{\prime}(s)>0$ for $u_{1}^{0} \leq$ $s<u_{1}^{*}$. If $u_{1}(x)$ reaches the critical value $u_{1}^{*}$, then equation (2.1) becomes degenerate. Figure 2 represents a situation where the function $F$ and coefficient $\alpha$ are such that $G_{\alpha}$ defined above satisfies those hypothesis with values $u_{1}^{0}$ and $u_{1}^{*}$.

We study in this section the problem (2.1), (2.2) under the following hypothesis:

$$
\begin{array}{ll}
\mathcal{H}_{0}- & F \text { is of class } C^{(2, \delta)} \text { on bounded intervals, } \\
\mathcal{H}_{1}- & \exists u_{1}^{0}>0 \text { such that } G_{\alpha}\left(u_{1}^{0}\right)=0, G_{\alpha}^{\prime}\left(u_{1}^{0}\right)>0, \\
\mathcal{H}_{2}- & \exists u_{1}^{*}>u_{1}^{0} \text { such that } G_{\alpha}^{\prime}\left(u_{1}^{*}\right)=0, G_{\alpha}^{\prime}(s)>0 \text { for } u_{1}^{0} \leq s<u_{1}^{*} .
\end{array}
$$

We use the notations $u_{1}^{0}$ and $u_{1}^{*}$ introduced above throughout the paper. We will show that classical solutions $u=\left(u_{1}, u_{2}\right)$ are such that $u_{1}^{0} \leq u_{1}(x)<u_{1}^{*}$ in $\Omega$ while for critical solution $\sup _{x \in \Omega} u_{1}(x)=u_{1}^{*}$ but then the measure of the critical set $D^{*}=\left\{x \in \Omega: u_{1}(x)=u_{1}^{*}\right\}$ is zero. 


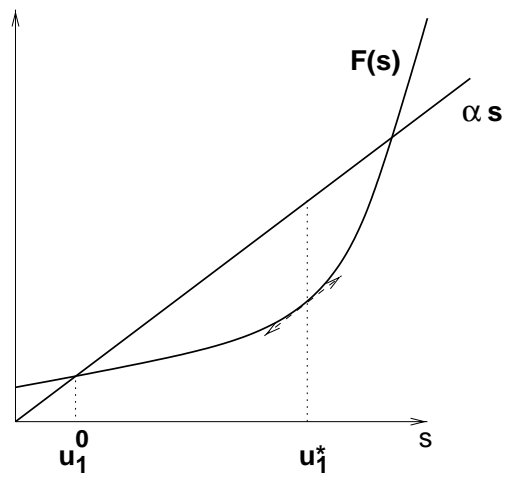

Figure 2. Representation of non-linearity $F(s)$, line $\alpha s$ and values $u_{1}^{0}$ and $u_{1}^{*}$.

Let $D$ be a subdomain in $\Omega$ with a smooth boundary. Integrating (2.1) over $D$, we obtain

$$
\int_{\partial D} G_{\alpha}^{\prime}\left(u_{1}\right) \frac{\partial u_{1}}{\partial n} \mathrm{~d} \sigma+\lambda^{2} \int_{D} F\left(u_{1}\right) \mathrm{d} x=0,
$$

where $n$ is the outer normal vector. This equality can be considered for a more general class of domains $D$ which have a finite perimeter. In this case, $\partial D$ should be understood as an essential boundary, $D$ as a set of points of density and the normal to the boundary is defined everywhere up to $(m-1)$-dimensional Hausdorff measure zero [12]. Moreover, the first integral is defined for functions with a bounded variation (i.e. from BV spaces) and in particular for $u_{1} \in C^{(0,1)}(\bar{\Omega})$. In what follows we use this equality only on functions $u_{1} \in C^{1}(\bar{\Omega})$ and for simplicity restrict ourselves to this class of functions. Hence, we will study functions $u_{1}$ satisfying the following condition:

Condition 1. $u_{1} \in C^{1}(\Omega)$, and for any subset $D \subset \Omega$ with a finite perimeter, $u_{1}$ satisfies (2.3).

Lemma 2.1. If $u_{1}$ satisfies Condition 1, then there exists a unique function $u_{2}(x) \in C^{(2, \delta)}(\bar{\Omega}), 0<\delta<1$ such that $u(x)=\left(u_{1}(x), u_{2}(x)\right)$ is a solution of (1.3)-(1.5).

Proof. Consider the problem

$$
\Delta u_{2}+\lambda^{2} F\left(u_{1}(x)\right)=0,\left.u_{2}\right|_{\partial \Omega}=0
$$

Since the function $F\left(u_{1}(x)\right)$ is Hölder continuous, then $u_{2}(x) \in C^{(2, \delta)}(\bar{\Omega})$ for some positive $\delta$ and for any subdomain $D$ of $\Omega$ with a finite perimeter:

$$
\int_{\partial D} \frac{\partial u_{2}}{\partial n} \mathrm{~d} \sigma+\lambda^{2} \int_{D} F\left(u_{1}\right) \mathrm{d} x=0 .
$$

We show now that it implies $G_{\alpha}\left(u_{1}(x)\right) \equiv u_{2}(x)$ in $\bar{\Omega}$. Denote $v(x)$ the function defined in $\bar{\Omega}$ by $v(x)=$ $u_{2}(x)-G_{\alpha}\left(u_{1}\right)$. Function $v$ belongs to $C^{1}(\bar{\Omega})$ and from $(2.3)$ and $(2.4)$ we obtain

$$
\int_{\partial D} \frac{\partial v}{\partial n} \mathrm{~d} \sigma=0
$$

We show that $\nabla v$ is identically zero in $\bar{\Omega}$. Suppose that it is not so. Then there exists some $x_{0} \in \Omega$ such that $v\left(x_{0}\right) \neq 0$ and $\nabla v\left(x_{0}\right) \neq 0$. Let for certainty $v\left(x_{0}\right)$ be positive. Consider the domain $D \subset \Omega$ where $v(x)>v\left(x_{0}\right)$. If the domain $D$ has a finite perimeter, then the integral $\int_{\partial D} \frac{\partial v}{\partial n} \mathrm{~d} \sigma$ is defined and negative since $\left.\frac{\partial v}{\partial n}\right|_{\partial D} \leq 0$ and $\left.\frac{\partial v}{\partial n}\right|_{x=x_{0}}<0$ which contradicts (2.5). If its perimeter is not finite, then we can choose $x_{1}$ close to $x_{0}$ such that $\nabla v\left(x_{1}\right) \neq 0$ and the set $D_{1}=\left\{x: v(x)>v\left(x_{1}\right)\right\}$ has a finite perimeter. Indeed, since $v(x)$ has a bounded 
variation, then the integral $\int_{-\infty}^{+\infty} P\left(E_{u}\right) d u$, where $P\left(E_{u}\right)$ is the perimeter of the set $E_{u}=\{x: v(x)>u\}$ is bounded [3]. The lemma is proved.

Proposition 2.2. Let $w(x)$ satisfy Condition 1, $\left.w(x)\right|_{\partial \Omega}=u_{1}^{0}$. If $w(x) \geq u_{1}^{0}$ in $\Omega$, then

$$
G_{\alpha}^{\prime}\left(u_{1}^{0}\right)>0
$$

and

$$
G_{\alpha}^{\prime}(w(x)) \geq 0, x \in \bar{\Omega}
$$

Proof. Let $D=\Omega$. The second integral in (2.3) is positive. Since $\partial u / \partial n \leq 0$, we obtain (2.6). Suppose that the inequality (2.7) does not hold and for some $x_{0} \in \Omega$,

$$
G_{\alpha}^{\prime}\left(w\left(x_{0}\right)\right)<0
$$

Since on the boundary $\partial \Omega$

$$
G_{\alpha}^{\prime}(w(x))>0
$$

then there exists a domain $D \subset \Omega$ such that

$$
G_{\alpha}^{\prime}(w(x))<0, \quad x \in D
$$

and

$$
G_{\alpha}^{\prime}(w(x))=0, \quad x \in \partial D .
$$

If the boundary of the domain $D$ is smooth, then we can use the formula (2.3) directly. The first integral in the left hand-side equals zero, while the second one is positive. This contradiction proves (2.7).

In the general case, we should consider sets with finite perimeters. We introduce the family of sets

$$
E_{u}=\{x: w(x)>u\}
$$

and denote by $P\left(E_{u}\right)$ the perimeter of the set $E_{u}$. Since the function $w(x)$ has a bounded variation, the integral

$$
I=\int_{-\infty}^{+\infty} P\left(E_{u}\right) \mathrm{d} u
$$

is bounded (see [3]). The value of $w(x)$ on the boundary of the domain $D$ can be found from $(2.8)$. Since $w(x)$ is continuous, its value is constant on each connected component of the boundary. Without loss of generality we can suppose that $D$ is connected and has interior points. Otherwise we take one of the connected components. Let $w(x)=u_{1}^{*}, x \in \partial D$. Suppose that the perimeter $P\left(E_{u_{1}^{*}}\right)$ is bounded. Then the Green's formula is valid for functions of bounded variations and we have

$$
\int_{\partial^{*} D} G_{\alpha}^{\prime}(w) \frac{\partial w}{\partial n} \mathrm{~d} \sigma+\lambda^{2} \int_{D^{*}} F(w) \mathrm{d} x=0
$$

where $\partial^{*} D$ is the essential boundary and $D^{*}$ is the set of density points of the set $D$ (see [13]). As above we obtain a contradiction since the first integral in (2.9) is zero while the second is positive.

Suppose now that $P\left(E_{u_{1}^{*}}\right)$ is not bounded. Since the integral $I$ is bounded, then there exists a sequence $\left\{u_{n}\right\}$, $u_{n} \rightarrow u_{1}^{*}$ such that $P\left(E_{u_{n}}\right)$ is bounded. Then

$$
\int_{\partial^{*} E_{u_{n}}} G_{\alpha}^{\prime}(w) \frac{\partial w}{\partial n} \mathrm{~d} \sigma+\lambda^{2} \int_{E_{u_{n}}^{*}} F(w) \mathrm{d} x=0 .
$$


Since $w(x)$ is continuous, then the lower limit of the second integral in (2.10) is positive. We show that the sequence $\left\{u_{n}\right\}$ can be chosen such that the integral

$$
J\left(u_{n}\right)=\int_{\partial^{*} E_{u_{n}}} G_{\alpha}^{\prime}(w) \frac{\partial w}{\partial n} \mathrm{~d} \sigma
$$

tends to zero as $n \rightarrow \infty$. This contradiction will prove the proposition.

Since $|\partial u / \partial n|$ is uniformly bounded and

$$
\left|G_{\alpha}^{\prime}(w)\right| \leq k\left|w-u_{1}^{*}\right|
$$

then

Here $k$ and $k_{1}$ are some constants. Suppose that

$$
\left|J\left(u_{n}\right)\right| \leq k_{1}\left|u_{n}-u_{1}^{*}\right| P\left(E_{u_{n}}\right) .
$$

$$
|J(u)| \geq \epsilon>0
$$

for $u$ in some neighborhood of $u_{1}^{*}$. Then

$$
P\left(E_{u}\right) \geq \frac{\epsilon}{k_{1}\left|u-u_{1}^{*}\right|}
$$

and the integral $I$ is not bounded. Thus there exists a sequence $\left\{u_{n}\right\}$ for which the integral $J\left(u_{n}\right)$ tends to zero. The proposition is proved.

Remark 2.3. The inequality (2.7) can be proved for a wider class of functions. Let $\left(u_{1}(x), u_{2}(x)\right)$ be a solution of the problem (1.3)-(1.5) such that $u_{1}$ is a continuous function and its first derivatives are uniformly bounded outside of the critical set where $u_{1}(x)=u_{1}^{*}$ and it is Lipschitz continuous in $\bar{\Omega}$. Suppose that there exists a domain $D \subset \Omega$ such that

$$
u_{1}(x)=u_{1}^{*}, x \in \partial D, \quad u_{1}(x)>u_{1}^{*}, x \in D .
$$

Then we can approximate $u_{1}^{*}$ by a sequence $\left\{u_{n}\right\}, u_{n}<u_{1}^{*}, u_{n} \rightarrow u_{1}^{*}$ such that the domains $E_{u_{n}}=\{x \in \Omega$ : $\left.u_{1}(x)>u_{n}\right\}$ have finite perimeter. The function $u_{1}(x)$ has continuous first derivatives in a neighborhood of each point $x \in \partial E_{u_{n}}$. The first integral in the equality (2.10) (with $w=u_{1}$ ) will converge to 0 , while the second integral to a positive constant. This contradiction proves that $u_{1}(x) \leq u_{1}^{*}$.

We introduce now the following hypothesis:

$$
\mathcal{H}_{3}-F^{\prime \prime}\left(u_{1}^{*}\right) \neq 0 \text {. }
$$

Proposition 2.4. Suppose that $\mathcal{H}_{0}-\mathcal{H}_{3}$ are satisfied. Let $u(x)=\left(u_{1}(x), u_{2}(x)\right)$ be a solution of the problem (1.3)-(1.5) and $u_{2}(x) \in C^{2}(\bar{\Omega})$. If

$$
\frac{F\left(u_{1}\right)-F\left(u_{1}^{*}\right)}{u_{1}-u_{1}^{*}} \geq \alpha, u_{1} \geq u_{1}^{0}, u_{1} \neq u_{1}^{*}
$$

then the first derivatives of the function $u_{1}(x)$ are bounded in $\bar{\Omega}$ uniformly outside of the critical set $D^{*}=$ $\left\{x: u_{1}(x)=u_{1}^{*}\right\}$ and $u_{1}(x)$ is Lipschitz continuous in $\bar{\Omega}$. The m-dimensional Hausdorff measure of the set $D^{+}=\left\{x: u_{1}(x) \geq u_{1}^{*}\right\}$ is zero. If this set is not empty, then the first derivatives of $u_{1}(x)$ are not continuous.

Proof. The boundedness of the derivatives $\frac{\partial u_{1}}{\partial x_{i}}$ easily follows from (1.3) for any $x$ that does not belong to $D^{*}$. We show that the derivatives remain bounded as $x \rightarrow D^{*}$. We note that from (2.11) it follows that

$$
F\left(u_{1}\right) \geq \alpha\left(u_{1}-u_{2}^{*}\right), u_{1} \geq u_{1}^{0}, u_{1} \neq u_{1}^{*}
$$

where $u_{2}^{*}=u_{1}^{*}-\frac{1}{\alpha} F\left(u_{1}^{*}\right)>0$. Hence the equation $F\left(u_{1}\right)=\alpha\left(u_{1}-u_{2}\right)$ does not have solutions for $u_{2}>u_{2}^{*}$. Consequently, $u_{2}(x) \leq u_{2}^{*}$ in $\bar{\Omega}$. 
Using the Taylor expansion:

$$
F\left(u_{1}\right)=F\left(u_{1}^{*}\right)+\alpha\left(u_{1}-u_{1}^{*}\right)+\frac{1}{2} F^{\prime \prime}\left(u_{1}^{*}\right)\left(u_{1}-u_{1}^{*}\right)^{2}\left(1+O\left(\left|u_{1}-u_{1}^{*}\right|\right)\right),
$$

we obtain from (1.3):

$$
u_{1}^{*}-u_{1}= \pm \sqrt{\frac{2 \alpha}{\left|F^{\prime \prime}\left(u_{1}^{*}\right)\right|}} \sqrt{\frac{u_{2}^{*}-u_{2}}{1+O\left(\left|u_{1}-u_{1}^{*}\right|\right)}}
$$

On the other hand, differentiating (1.3) with respect to $x_{i}$, we have:

$$
\left(1-\frac{1}{\alpha} F^{\prime}\left(u_{1}\right)\right) \frac{\partial u_{1}}{\partial x_{i}}=\frac{\partial u_{2}}{\partial x_{i}} .
$$

Taking into account that $F^{\prime}\left(u_{1}^{*}\right)=\alpha$, we have also another expansion:

$$
1-\frac{1}{\alpha} F^{\prime}\left(u_{1}\right)=-\frac{1}{\alpha} F^{\prime \prime}\left(u_{1}^{*}\right)\left(u_{1}-u_{1}^{*}\right)\left(1+O\left(\left|u_{1}-u_{1}^{*}\right|\right)\right) .
$$

From (2.13)-(2.15) we have:

$$
\begin{aligned}
\frac{\partial u_{1}}{\partial x_{i}} & =\frac{\partial u_{2}}{\partial x_{i}} \frac{1}{\frac{1}{\alpha} F^{\prime \prime}\left(u_{1}^{*}\right)\left(u_{1}^{*}-u_{1}\right)\left(1+O\left(\left|u_{1}-u_{1}^{*}\right|\right)\right)} \\
& = \pm \sqrt{\frac{\alpha}{2\left|F^{\prime \prime}\left(u_{1}^{*}\right)\right|}} \frac{\frac{\partial u_{2}}{\partial x_{i}}}{\sqrt{u_{2}^{*}-u_{2}}}\left(1+O\left(\left|u_{1}-u_{1}^{*}\right|\right)\right) .
\end{aligned}
$$

It remains to use the estimation (see [7]):

$$
\left|\frac{\partial u_{2}}{\partial x_{i}}\right| \leq 2 \sqrt{\sup \mid \frac{\partial^{2} u_{2}}{\partial x_{i}^{2}}} \mid \sqrt{u_{2}^{*}-u_{2}}
$$

Thus we have proved that the first derivatives $\frac{\partial u_{1}}{\partial x_{i}}$ are uniformly bounded outside the critical set $D^{*}$. We show now that $u_{1}(x)$ satisfies a Lipschitz condition in $\bar{\Omega}$. For any $x_{0}, x \in \bar{\Omega} \backslash D^{*}$ the estimation

$$
\left|u_{1}(x)-u_{1}\left(x_{0}\right)\right| \leq K\left|x-x_{0}\right|
$$

follows from the uniform boundedness of the derivatives. Taking the limit in the above expression as $x_{0}$ tends to $x_{0}^{*} \in D^{*}$, we obtain that $u_{1}(x)$ satisfies a Lipschitz condition and has a bounded variation. From Remark 2.3 it follows that $u_{1}(x) \leq u_{1}^{*}$ in $\bar{\Omega}$ and the same method allows to prove that $\mu\left(D^{+}\right)=0$ where $\mu\left(D^{+}\right)$denotes the $m$-dimensional Hausdorff measure of the set $D^{+}$.

Suppose that the set $D^{*}$ is not empty and $u_{1}(x) \in C^{1}(\bar{\Omega})$. Let $x_{0} \in D^{*}$ and choose $D$ in $(2.3)$ to be a small ball with the center $x_{0}$ and radius $r$. Then the second integral in $(2.3)$ is equivalent to $\lambda^{2}\left(F\left(u_{1}^{*}\right)+\varepsilon(r)\right) \mu(D)$ where $\varepsilon(r) \rightarrow 0$ as $r \rightarrow 0$, while the first integral is equivalent to $\phi(r) \mu(D)$ where $\phi(r) \rightarrow 0$ as $r \rightarrow 0$. For $r$ sufficiently small, we obtain:

$$
0<\lambda^{2}\left(F\left(u_{1}^{*}\right)+\varepsilon(r)-\phi(r)\right) \mu(D)<\int_{\partial D} G_{\alpha}^{\prime}\left(u_{1}\right) \frac{\partial u_{1}}{\partial n} \mathrm{~d} \sigma+\lambda^{2} \int_{D} F\left(u_{1}\right) \mathrm{d} x=0 .
$$

Note that if $u$ is only Lipschitz continuous, the term $\int_{\partial D} G_{\alpha}^{\prime}\left(u_{1}\right) \frac{\partial u_{1}}{\partial n} d \sigma$ is not defined. This contradiction proves the proposition. 
Remark 2.5. In the case of a convex non-linearity (2.11) is satisfied.

We use (2.11) to consider values of $u_{1}$ greater than $u_{1}^{*}$ (see Remark 2.3).

In the remaining part of this section, we assume

$\mathcal{H}_{4}{ }^{-} \quad$ on each interval of monotonicity of the function $t=G_{\alpha}(s)$ the inverse function $s=H_{\alpha}(t)$ is uniformly Hölder continuous with an exponent $\gamma, 0<\gamma<1$.

Lemma 2.6. Let $\left(u_{1}(x), u_{2}(x)\right)$ be a solution of the problem (1.3)-(1.5) defined in all $\bar{\Omega}, u_{1}(x)$ be bounded in $\bar{\Omega}$ and $u_{2} \in W^{2, p}$ for some $p>1$. Then the critical set $D^{*}=\left\{x: u_{1}(x)=u_{1}^{*}\right\}$ does not have interior points and $u_{1}(x) \in C^{(\delta)}(\bar{\Omega}), u_{2}(x) \in C^{(2, \delta)}(\bar{\Omega})$ for some $\delta$.

Proof. Since $u_{1}(x) \in L^{p}(\Omega)$ for any $p>1$, then there exists a solution $v_{2} \in W^{2, p}(\Omega)$ of (1.4). By uniqueness of solutions, $v_{2}=u_{2}$ and by the embedding theorems, $u_{2}(x) \in C^{(\sigma)}(\bar{\Omega})$ for some $\sigma>0$. Then from (1.3) $u_{1}(x) \in C^{(\gamma \sigma)}(\bar{\Omega})$ and from $(1.4) u_{2}(x) \in C^{(2, \gamma \sigma)}(\bar{\Omega})$. From Propositions 2.4 and 2.2 it follows that $u_{1}(x) \leq u_{1}^{*}$ and $u_{2}(x) \leq u_{2}^{*}$ in $\bar{\Omega}$.

On the critical set, $u_{2}(x) \equiv G_{\alpha}\left(u_{1}^{*}\right)$. If it has interior points, then $\Delta u_{2}=0$ on it and (1.4) gives a contradiction. The lemma is proved.

From the results above we obtain the following theorem:

Theorem 2.7. Let $\mathcal{H}_{0}-\mathcal{H}_{4}$ and (2.11) be satisfied.

If $u(x)=\left(u_{1}(x), u_{2}(x)\right)$ is a solution of the problem (1.3)-(1.5) such that $u_{1}(x)$ is bounded and $u_{2} \in W^{2, p}(\Omega)$ for some $p>1$, then $u_{1}^{0} \leq u_{1}(x) \leq u_{1}^{*}, u_{1}(x)$ has bounded first derivatives outside of the critical set $D^{*}=\{x$ : $\left.u_{1}(x)=u_{1}^{*}\right\}$ and it is Lipschitz continuous in $\bar{\Omega}, u_{2}(x) \in C^{(2, \delta)}(\bar{\Omega})$ for some $\delta>0$. If the critical set $D^{*}$ is not empty, then $u_{1}(x)$ does not belong to $C^{1}(\bar{\Omega})$.

Definition 2.8. We will say that $\left(u_{1}, u_{2}\right)$ is a critical solution of (1.3)-(1.5) if the critical set $D^{*}=\left\{x: u_{1}(x)=\right.$ $\left.u_{1}^{*}\right\}$ is not empty.

In the end of this section we show that a critical solution can be obtained as a limit of classical solutions.

Theorem 2.9. Let $\left(u_{1}^{n}, u_{2}^{n}\right)$ be solutions of the problems (1.3),(1.5) with $\lambda=\lambda_{n}$ and $\sup _{x} u_{1}^{n}(x)<u_{1}^{*}$. Suppose that $\lambda_{n} \rightarrow \lambda^{*}$ and

$$
\lim _{n \rightarrow \infty} \sup _{x \in \bar{\Omega}} u_{1}^{n}(x)=u_{1}^{*} .
$$

Then there exists a solution $\tilde{u}_{1}(x) \in C^{(\delta)}(\bar{\Omega}), \tilde{u}_{2}(x) \in C^{(2, \delta)}(\bar{\Omega})$ of the problem (1.3)-(1.5) with $\lambda=\lambda^{*}$ and there is a subsequence of $u_{i}^{n}(x)$ converging to $\tilde{u}_{i}(x)$ uniformly in $\Omega$.

Proof. It is sufficient to note that the following estimations are independent of n:

$$
\begin{aligned}
& \left\|u_{1}^{n}\right\|_{L^{p}} \leq M_{1},\left\|u_{2}^{n}\right\|_{W^{2, p}} \leq M_{2},\left\|u_{2}^{n}\right\|_{C^{(\sigma)}} \leq M_{3} \\
& \left\|u_{1}^{n}\right\|_{C^{(\gamma \sigma)}} \leq M_{4},\left\|u_{2}^{n}\right\|_{C^{(2, \gamma \sigma)}} \leq M_{5}
\end{aligned}
$$

The first estimation is obvious. Each of the other estimations uses the previous one. Moreover, the second uses (1.3) and (1.4); the third, embedding theorems; the fourth, (1.3); the fifth, (1.4). The theorem is proved.

\section{EXISTENCE OF SOLUTIONS}

In the first part of this section $\lambda$ is a fixed parameter and we study the asymptotics for large $\alpha$ (convergence towards the problem of heat explosion in homogeneous medium as the heat exchange between the two phases becomes infinite), while from Lemma 3.3 until the end of the section, $\alpha$ is arbitrary and we study the existence of solutions to (1.3)-(1.5) relatively to the size parameter $\lambda$.

We show first that for large $\alpha$ solutions of the problem $(2.1),(2.2)$ converge to solutions of the problem

$$
\Delta w+\lambda^{2} F(w)=0,\left.\quad w\right|_{\partial \Omega}=0 .
$$


We assume that $\mathcal{H}_{0}-\mathcal{H}_{2}$ are satisfied.

Theorem 3.1. Suppose that the boundary $\partial \Omega$ belongs to the class $C^{(2, \delta)}$. If there exists a solution $w(x)$ of the problem (3.1) and the linearized problem

$$
\Delta u+\lambda^{2} F^{\prime}(w(x)) u=0,\left.\quad u\right|_{\partial \Omega}=0
$$

has only zero solution, then the problem (2.1), (2.2) has a solution for all $\alpha \geq \alpha_{0}$, where $\alpha_{0}$ is sufficiently large, and it converges to $w(x)$ in $C^{(2)}(\bar{\Omega})$ as $\alpha \rightarrow \infty$.

Proof. Denote by $u_{1}^{0, \alpha}$ a positive solution of $G_{\alpha}(s)=0$. Hence $u_{1}^{0, \alpha}=\frac{1}{\alpha} F\left(u_{1}^{0, \alpha}\right)$ and since $F$ is bounded on any bounded interval of $\mathbb{R}_{+}$, then $u_{1}^{0, \alpha}$ tends to zero as $\alpha \rightarrow \infty$. If $u_{1}$ is a classical solution of $(2.2), v=u_{1}-u_{1}^{0, \alpha}$ is a solution of the equivalent problem:

$$
\begin{gathered}
G_{\alpha}^{\prime}\left(v+u_{1}^{0, \alpha}\right) \Delta v+G_{\alpha}^{\prime \prime}\left(v+u_{1}^{0, \alpha}\right)|\nabla v|^{2}+\lambda^{2} F\left(v+u_{1}^{0, \alpha}\right)=0, \\
\left.v\right|_{\partial \Omega}=0 .
\end{gathered}
$$

We consider a small neighborhood of the function $w(x)$ in the norm $C^{(2, \delta)}(\bar{\Omega})$, where $w$ is a solution of (3.1) that satisfies the hypothesis of the theorem. In this neighborhood

$$
G_{\alpha}^{\prime}\left(v+u_{1}^{0, \alpha}\right) \geq \epsilon>0
$$

for $\alpha$ sufficiently large. Then equation (3.3) can be written as

$$
\Delta v+\frac{G_{\alpha}^{\prime \prime}\left(v+u_{1}^{0, \alpha}\right)|\nabla v|^{2}+\lambda^{2} F\left(v+u_{1}^{0, \alpha}\right)}{G^{\prime}\left(v+u_{1}^{0, \alpha}\right)}=0 .
$$

Consider the operator

$$
A(v)=L v+B(v),
$$

acting from $C_{0}^{(2, \delta)}(\bar{\Omega})$ to $C^{(\delta)}(\bar{\Omega})$, where $L v=\Delta v$ and

$$
B(v)=\frac{G_{\alpha}^{\prime \prime}\left(v+u_{1}^{0, \alpha}\right)|\nabla v|^{2}+\lambda^{2} F\left(v+u_{1}^{0, \alpha}\right)}{G_{\alpha}^{\prime}\left(v+u_{1}^{0, \alpha}\right)} .
$$

Let $U$ be a bounded domain in $C_{0}^{(1, \delta)}(\bar{\Omega}), 0 \leq \delta<1$ such that all functions from $U$ satisfy (3.5). Then the operator $B(v)$ is bounded as acting from $U$ to $C^{(\delta)}(\bar{\Omega})$. Since the operator

$$
L^{-1}: C^{(\delta)}(\bar{\Omega}) \rightarrow C_{0}^{(1, \delta)}(\bar{\Omega})
$$

is compact, then $L^{-1} A(v)=v+K(v)$, where $K(v)=L^{-1} B(v)$, is a compact operator from $U$ to $C_{0}^{(1, \delta)}(\bar{\Omega})$. Denote $\mu=\frac{1}{\alpha}$ and consider the parametrized operator

$$
A_{\mu}(v)=L v+B_{\mu}(v)
$$

where

and put

$$
B_{\mu}(v)=\left(-\mu F^{\prime \prime}\left(v+u_{1}^{(0, \alpha)}\right)|\nabla v|^{2}+\lambda^{2} F\left(v+u_{1}^{(0, \alpha)}\right)\right) /\left(1-\mu F^{\prime}\left(v+u_{1}^{(0, \alpha)}\right)\right)
$$

$$
M_{\mu}(v)=L^{-1}\left(A_{\mu}(v)\right)=v+K_{\mu}(v)
$$


with

We have

$$
K_{\mu}(v)=L^{-1} B_{\mu}(v)
$$

$$
M_{0}(v)=L^{-1}\left(\Delta v+\lambda^{2} F(v)\right) .
$$

Then

$$
M_{0}(w)=0
$$

and

$$
M_{0}^{\prime}(w) u=L^{-1}\left(\Delta u+\lambda^{2} F^{\prime}(w) u\right) .
$$

Thus the linearized operator does not have zero eigenvalue and the index of the stationary point $w$ (topological degree computed on a small neighborhood of $w$ ) is defined and equals $(-1)^{\nu}$ where $\nu$ is the number of positive eigenvalues of the operator $M_{0}^{\prime}(w)$. Hence the index of $w$ is different from zero.

We note that

$$
\sup _{v \in U}\left\|B_{\mu}(v)-B_{0}(v)\right\|^{(\delta)} \rightarrow 0, \quad \text { as } \mu \rightarrow 0,
$$

where $\|\cdot\|^{(\delta)}$ is the norm in $C^{(\delta)}(\bar{\Omega})$. So if

$$
M_{0}(v) \neq 0, \quad v \in \partial U
$$

then

for $\mu$ sufficiently small, and

$$
M_{\mu}(v) \neq 0, \quad v \in \partial U
$$

$$
\gamma\left(M_{\mu}, U\right)=\gamma\left(M_{0}, U\right)
$$

where $\gamma$ denotes the degree of the operator over domain $U$. As a set $U$ we choose a small neighborhood of an isolated solution $v=w$ of the equation $M_{0}(v)=0$ with nonzero index. Then (3.8) is satisfied and thus for $\mu=\frac{1}{\alpha}$ sufficiently small there is a solution $v_{\mu}$ of the equation $M_{\mu}(v)=0$ in $U$. By construction, $v_{\mu}$ belongs to $C_{0}^{(1, \delta)}(\bar{\Omega})$. It can be easily verified that $v_{\mu}$ is in $C_{0}^{(2, \delta)}(\bar{\Omega})$. Indeed, solutions $\tilde{u}$ of the linear problems, $L \tilde{u}+B_{\mu}\left(v_{\mu}\right)=0$ belong to $C_{0}^{(2, \delta)}(\bar{\Omega})$. Since $\tilde{u}=L^{-1} B_{\mu}\left(v_{\mu}\right)$ and $v_{\mu}=L^{-1} B_{\mu}\left(v_{\mu}\right)$, then $v_{\mu}=\tilde{u}$. Convergence of $v_{\mu}$ to $w$ in $C^{(2)}(\bar{\Omega})$ follows from this. The theorem is proved.

Theorem 3.2. Suppose that the problem (3.1) does not have solutions in a bounded domain $G \subset C_{0}^{(2, \delta)}(\bar{\Omega})$. Then for all $\alpha$ sufficiently large the problem (2.1), (2.2) does not have solutions in $G$.

Proof. Suppose that there is a sequence $\left\{\alpha_{n}\right\}, \alpha_{n} \rightarrow \infty$ such that the problems $(2.1,2.2)$ with $\alpha=\alpha_{n}$ have solutions in the domain $G$. Then the equation

$$
M_{\mu_{n}}(v)=0
$$

has a solution $v_{n} \in G$. Here $\alpha_{n}=1 / \mu_{n}$ and the operator $M_{\mu}$ is the same as in the proof of the previous theorem. Then

$$
v_{n}=-K_{\mu_{n}}\left(v_{n}\right)
$$

and we can choose a subsequence from the sequence $\left\{v_{n}\right\}$ converging to some $v_{0} \in G$. Then $M_{0}\left(v_{0}\right)=0$ and $v_{0}$ is a solution of the problem (3.1). This contradiction proves the theorem.

Now we consider $\alpha$ fixed and study the continuous branches of solutions depending on the size of the domain. In the following lemma we suppose that $\lambda$ denotes the diameter of the domain.

Lemma 3.3. If the diameter $\lambda$ of the domain $\Omega_{\lambda}$ is sufficiently small, then there exists a solution of the problem (1.3)-(1.5). 
Proof. We consider the degenerate parabolic system in the domain $\Omega_{\lambda}$, that is the evolution problem corresponding to $(1.1),(1.2),(1.5)$

$$
\begin{aligned}
& \frac{\partial u_{1}}{\partial t}=F\left(u_{1}\right)-\alpha\left(u_{1}-u_{2}\right), \\
& \frac{\partial u_{2}}{\partial t}=\Delta u_{2}-\alpha\left(u_{2}-u_{1}\right)
\end{aligned}
$$

and construct a lower and upper functions for it. As a lower function we take

$$
\phi(x)=\left(\phi_{1}(x), \phi_{2}(x)\right), \quad \phi_{1}(x) \equiv u_{1}^{0}, \phi_{2}(x) \equiv 0 \text { in } \Omega .
$$

We proceed now to construction of an upper function. Without loss of generality we can assume that $0 \in \Omega$. We put

$$
\psi(x)=-a|x|^{2}+b, \quad|x|=\sqrt{x_{1}^{2}+\ldots+x_{m}^{2}}
$$

where $a$ and $b$ are positive constants such that $b / a>\lambda^{2}$. Then $\psi(x)>\phi_{2}(x) \equiv 0$ in $\bar{\Omega}$. We define the function $v(x)=\left(v_{1}(x), v_{2}(x)\right)$ by the equalities

$$
\begin{gathered}
v_{2}(x)=\psi(x), \\
F\left(v_{1}(x)\right)-\alpha\left(v_{1}(x)-v_{2}(x)\right)=0 .
\end{gathered}
$$

Since $F^{\prime}\left(u_{1}^{0}\right)<\alpha$, then for $b=\max _{x \in \Omega} v_{2}(x)$ sufficiently small, $v_{1}(x)$ can be found from (3.11) and $v_{1}(x)>$ $\phi_{1}(x)$. Moreover

We have further

$$
\max _{x}\left(v_{1}(x)-u_{1}^{0}\right) \rightarrow 0, \quad \text { as } b \rightarrow 0 .
$$

$$
\Delta v_{2}-\alpha\left(v_{2}-v_{1}\right)=-2 m a+F\left(v_{1}(x)\right)
$$

If

$$
a>F\left(u_{1}^{0}\right) / 2 m,
$$

then the right-hand side of the last equality is negative for $b$ sufficiently small. Thus $v(x)$ is an upper function.

Solution of the system (3.9), (3.10) with the initial condition $u(x, 0)=\phi(x)$ and the boundary condition $\left.u_{2}\right|_{\partial \Omega}=0$ increases in time and it is bounded from above by the function $v(x)$. Then it converges to a stationary solution. The lemma is proved.

Remark 3.4. From the proof of the lemma it follows that there exists a stationary solution $u(x)$ of $(1.3)-(1.5)$ such that

$$
\max _{\bar{\Omega}}\left|u_{1}(x)-u_{1}^{0}\right| \rightarrow 0, \max _{\bar{\Omega}}\left|u_{2}(x)\right| \rightarrow 0, \text { as } \lambda \rightarrow 0 .
$$

Remark 3.5. The use of upper and lower functions for the evolution problem is equivalent to the use of upper and lower solutions for the stationary equations. Proof of Theorem 4.1 in the next section relies on an iterative scheme based on upper and lower solutions.

We denote $E_{0}$ the space of functions $w(x)=\left(w_{1}(x), w_{2}(x)\right)$ such that $w_{1}(x) \in C^{(\delta)}(\bar{\Omega}), w_{2}(x) \in C_{0}^{(2, \delta)}(\bar{\Omega})$, and $E_{1}=E_{0} \times \mathbb{R}$.

Lemma 3.6. Let $K$ be a compact set in a Banach space $E$ and $K_{0}$ be one of its connected component. For any $\delta>0$ sufficiently small, there exists a domain $G_{\delta}$ such that

$$
K_{0} \subset G_{\delta}, \quad K \cap \partial G_{\delta}=\emptyset, \operatorname{dist}\left(K_{0}, \partial G_{\delta}\right) \leq \delta .
$$


Proof. For any $u_{0} \in K$ we denote by $B_{\sigma}\left(u_{0}\right)$ the sphere $\left\|u-u_{0}\right\|<\sigma$ and

$$
G_{\sigma}=\cup_{u_{0} \in K} B_{\sigma}\left(u_{0}\right) .
$$

Let $G_{\sigma}^{0}$ be a connected component of the domain $G_{\sigma}$ containing the set $K_{0}$. Then

$$
K_{0} \subset G_{\sigma}^{0} \text {, and by construction } K \cap \partial G_{\sigma}^{0}=\emptyset .
$$

It is sufficient to show that for $\sigma$ sufficiently small

$$
\operatorname{dist}\left(K_{0}, \partial G_{\sigma}^{0}\right) \leq \delta
$$

for any given positive $\delta$.

Suppose that there exists $\delta>0$ such that (3.13) does not take place for any $\sigma$. We consider a sequence $\left\{\sigma_{n}\right\}$ decreasing and converging to zero, and the corresponding sequence of domains $G_{\sigma_{n}}^{0}$. Then

$$
\begin{gathered}
\bar{G}_{\sigma_{n}}^{0} \subset G_{\sigma_{m}}^{0}, \quad n>m, \\
K_{1}=\cap_{n=1,2, \ldots} \bar{G}_{\sigma_{n}}^{0} \subset K .
\end{gathered}
$$

The set $K_{1}$ is closed. Moreover, since (3.13) does not hold, then there exists $u_{1} \in K_{1}$ such that $u_{1} \notin K_{0}$.

We show that the set $K_{1}$ is connected. It will give a contradiction with the assumption that $K_{0}$ is a connected component of the set $K$ and will prove the lemma. Suppose that $K_{1}$ is not connected. Then there exist two sets $K_{1}^{1}$ and $K_{1}^{2}$ such that

$$
K_{1}=K_{1}^{1} \cup K_{1}^{2}, \quad \bar{K}_{1}^{1} \cap K_{1}^{2}=\emptyset, \quad K_{1}^{1} \cap \bar{K}_{1}^{2}=\emptyset .
$$

Since the domain $G_{\sigma_{n}}^{0}$ is connected for any $\sigma_{n}$ in the considered sequence, then there exists a sequence $\left\{u_{n}\right\}$ such that

$$
u_{n} \in K_{1}^{2}, \operatorname{dist}\left(u_{n}, K_{1}^{1}\right) \rightarrow 0, n \rightarrow \infty .
$$

By virtue of the assumption that the set $K$ is compact, there is a subsequence of the sequence $\left\{u_{n}\right\}$ converging to some $u^{*} \in K_{1}$. Then $u^{*} \in \bar{K}_{1}^{1}$ and $u^{*} \in \bar{K}_{1}^{2}$. If $u^{*} \in K_{1}^{1}$, then $K_{1}^{1} \cap \bar{K}_{1}^{2} \neq \emptyset$, if $u^{*} \in K_{1}^{2}$, then $\bar{K}_{1}^{1} \cap K_{1}^{2} \neq \emptyset$. Hence the set $K_{1}$ is connected. The lemma is proved.

Lemma 3.7. Let $K$ be the set of solutions $w=\left(w_{1}, w_{2}\right)$ of the problem (1.3)-(1.5) in a ball $\|w\| \leq R$ in $E_{0}$ for $\lambda_{0} \leq \lambda \leq \lambda_{1}$. Suppose that

$$
\sup _{w \in K} \max _{x \in \Omega} w_{1}(x)<u_{1}^{*} .
$$

Then the set $K$ is compact.

Proof. Let $\left\{w^{(n)}\right\}$ be a sequence in $K$. We show that there is a converging subsequence of this sequence.

Let $A_{\lambda}(w)$ be the operator corresponding to the problem (1.3)-(1.5),

$$
A_{\lambda_{n}}\left(w^{(n)}\right)=0 .
$$

Without loss of generality we can assume that the sequence $\left\{\lambda_{n}\right\}$ converges to some $\tilde{\lambda}$.

Since the first components $w_{1}^{(n)}(x)$ of the vector-valued function $w^{(n)}(x)$ are bounded in $C^{(\delta)}(\bar{\Omega})$, then from the equation (1.4) we conclude that $w_{2}^{(n)}(x)$ is uniformly bounded in $C^{(2, \delta)}(\bar{\Omega})$. Then there exists a subsequence $w_{2}^{\left(n_{k}\right)}(x)$ converging in $C^{(2)}(\bar{\Omega})$ to some function $\tilde{w}_{2}(x)$. From (1.3) it follows that $w_{1}^{\left(n_{k}\right)}(x)$ converges to $\tilde{w}_{1}(x)$. Then the function $\tilde{w}(x)=\left(\tilde{w}_{1}(x), \tilde{w}_{2}(x)\right)$ is a solution of the equation

$$
A_{\tilde{\lambda}}(w)=0 .
$$


We show that $w^{\left(n_{k}\right)}(x)$ converges to $\tilde{w}(x)$ in $E_{0}$. We have

$$
\begin{aligned}
& z_{1}^{(k)}=z_{2}^{(k)}+\frac{1}{\alpha}\left(F\left(\tilde{w}_{1}(x)\right)-F\left(w_{1}^{\left(n_{k}\right)}(x)\right)\right) \\
& \Delta z_{2}^{(k)}-\alpha \tilde{\lambda}^{2}\left(z_{2}^{(k)}-z_{1}^{(k)}\right)=\alpha\left(\tilde{\lambda}^{2}-\lambda_{n_{k}}{ }^{2}\right)\left(w_{2}^{\left(n_{k}\right)}-w_{1}^{\left(n_{k}\right)}\right), \\
& \left.z_{2}^{(k)}\right|_{\partial \Omega}=0
\end{aligned}
$$

where

$$
z_{i}^{(k)}=\tilde{w}_{i}-w_{i}^{\left(n_{k}\right)}, \quad i=1,2 .
$$

By virtue of the Schauder estimates $z^{(k)}$ converges to 0 in $E_{0}$. The lemma is proved.

Corollary 3.8. The set $\mathcal{S}$ of solutions $\left(u_{1}, \lambda\right)$ of the problem

$$
\operatorname{div}\left(G_{\alpha}^{\prime}\left(u_{1}+u_{1}^{0}\right) \nabla u_{1}\right)+\lambda^{2} F\left(u_{1}+u_{1}^{0}\right)=0,\left.\quad u_{1}\right|_{\partial \Omega}=0
$$

such that

$$
\sup _{\left(u_{1}, \lambda\right) \in \mathcal{S}} \max _{x \in \Omega} u_{1}(x)<u_{1}^{*}
$$

is compact in any bounded closed set in $C_{0}^{2, \delta}(\bar{\Omega}) \times \mathbb{R}$ (i.e. for any closed and bounded set $\mathcal{B}$ in $C_{0}^{2, \delta}(\bar{\Omega}) \times \mathbb{R}$, $\mathcal{S} \cap \mathcal{B}$ is compact).

Lemma 3.9. For any $u_{1}^{0}<m<u_{1}^{*}$ there exists $\lambda_{0}$ such that for any solution $u(x)=\left(u_{1}(x), u_{2}(x)\right)$ of the problem (1.3)-(1.5) with $\lambda>\lambda_{0}$, we have $\max _{x} u_{1}(x)>m$.

Proof. Suppose that for any $\lambda$ there exists a solution such that $\max _{x} u_{1}(x) \leq m$. Then $\max _{x} u_{2}(x) \leq m-\frac{1}{\alpha} F(m)$. Consider the problem

$$
\Delta u_{2}+\lambda^{2} b=0,\left.\quad u_{2}\right|_{\partial \Omega^{\prime}}=0
$$

in a domain $\Omega^{\prime} \subset \Omega$, where $b$ is a positive constant such that $F\left(u_{1}\right) \geq b, u_{1}^{0} \leq u_{1} \leq u_{1}^{*}$. It has a unique positive solution $w_{\lambda}(x)$ and its maximum is a linear function of $\lambda^{2} b$. The function $\tilde{u}(x)$ equal to $w_{\lambda}(x)$ in $\Omega^{\prime}$ and 0 in $\Omega /$ $\Omega^{\prime}$ is a lower function for the problem (1.5), (1.6). For $\lambda$ sufficiently small $\tilde{u}(x)<u_{2}(x)$ in $\Omega^{\prime}$. For $\lambda$ sufficiently large $\max _{x \in \Omega^{\prime}} \tilde{u}(x)>m-\frac{1}{\alpha} F(m) \geq \max _{x \in \Omega} u_{2}(x)$. Hence there exists $\lambda=\lambda_{0}$ such that $\tilde{u}(x) \leq u_{2}(x)$ in $\Omega^{\prime}$ and $\tilde{u}\left(x_{0}\right)=u_{2}\left(x_{0}\right), x_{0} \in \Omega^{\prime}$. The function $v(x)=u_{2}(x)-\tilde{u}(x)$ satisfies the equation $\Delta v+\lambda_{0}^{2}\left(F\left(u_{1}\right)-b\right)=0$ and $v(x) \geq 0$ in $\Omega^{\prime}, v\left(x_{0}\right)=0$. This contradicts to the positiveness theorem for linear elliptic equations. The lemma is proved.

Theorem 3.10. Under the hypothesis of Theorem 2.7, there exists a connected set $M \subset E_{1}$ such that for any $(u(x), \lambda) \in M, u(x)$ is a solution of the problem (1.3)-(1.5), $\left(u_{1} \equiv u_{1}^{0}, u_{2} \equiv 0, \lambda=0\right) \in M$ and

$$
\sup _{(u, \lambda) \in M} \max _{x \in \Omega} u_{1}(x)=u_{1}^{*}
$$

Proof. As a set $M$ we take a connected set of pairs $(u(x), \lambda)$ in $E_{1}$ such that $u(x)$ is a solution of the problem $(1.3-1.5)$ and that $\left(u_{1} \equiv u_{1}^{0}, u_{2} \equiv 0, \lambda=0\right) \in M$. We recall that from Lemma 3.3 and the remark after the Lemma, if $\lambda$ is sufficiently small there exists a solution of (1.3)-(1.5) such that

$$
\max _{\bar{\Omega}}\left|u_{1}(x)-u_{1}^{0}\right| \rightarrow 0, \max _{\bar{\Omega}}\left|u_{2}(x)\right| \rightarrow 0, \quad \lambda \rightarrow 0 .
$$


Suppose that

$$
\sup _{(u, \lambda) \in M} \max _{x \in \Omega} u_{1}(x)<u_{1}^{*} .
$$

By virtue of Lemma 3.9 and of estimates of classical solutions, the set of solutions of the problem (3.17) is bounded in $C^{(2, \delta)}(\bar{\Omega}) \times \mathbb{R}$. Moreover, the projection on $C^{(2, \delta)}(\bar{\Omega}) \times \mathbb{R}$ of a connected set of solutions in $E_{1}$ of (1.3)-(1.5) is a connected set of solutions of (3.17) and reciprocally. From Lemma 3.6 and Corollary 3.8 it follows that a bounded domain $G \subset C^{(2, \delta)}(\bar{\Omega})$ can be chosen such that for any solution $\left.u(x)=\left(u_{1}(x), u_{2}(x)\right), \lambda\right)$ in $M, u_{1}(x)$ belong to it,

$$
\sup _{u_{1} \in G} \max _{x \in \Omega} u_{1}(x)<u_{1}^{*},
$$

and there are no solutions of the problem (3.17) on the boundary $\partial G$.

We use the topological degree arguments as in the proof of Theorem 3.1 to obtain a contradiction. We consider the operator:

$$
A_{\lambda}(v)=\Delta v+B_{\lambda}(v),
$$

acting from $C_{0}^{(2, \delta)}(\bar{\Omega})$ to $C^{(\delta)}(\bar{\Omega})$, where

$$
B_{\lambda}(v)=\frac{\left(G_{\alpha}^{\prime \prime}\left(v+u_{1}^{0, \alpha}\right)|\nabla v|^{2}+\lambda^{2} F\left(v+u_{1}^{0, \alpha}\right)\right)}{G_{\alpha}^{\prime}\left(v+u_{1}^{0, \alpha}\right)} .
$$

For $\lambda=0$ there exists a single solution $v=0$ of the equation $A_{0}(v)=0$ in the domain $G$. Since $A_{0}^{\prime}(0) u=\Delta u$, then the topological degree $\gamma\left(A_{0}, G\right)$ of the operator $A_{0}(v)$ over domain $G$ equals 1 (c.f. demonstration of Theorem 3.1). We recall that $A_{\lambda}(v) \neq 0$ for $v \in \partial G$ and $\lambda>0$. Hence $\gamma\left(A_{\lambda}, G\right)=\gamma\left(A_{0}, G\right) \neq 0$. On the other hand, by virtue of Lemma 3.9 there are no solutions of the equation $A_{\lambda}(v)=0$ in $G$ for $\lambda$ sufficiently large. Thus $\gamma\left(A_{\lambda}, G\right)=0$. this contradiction proves the theorem.

\section{Minimax Representation of the CRItical CONDition}

In this section we obtain critical conditions of existence of solutions of the problem $(2.1,2.2)$ in a minimax form. We will then show on an example how this minimax condition can be used to obtain approximate critical conditions of existence of solutions.

Theorem 4.1. Suppose that $\mathcal{H}_{0}-\mathcal{H}_{2}$ are satisfied and

$$
F^{\prime \prime}\left(u_{1}\right)>0, u_{1}^{0} \leq u_{1}<u_{1}^{*} .
$$

Then the inequality

$$
\frac{1}{\alpha} \geq \inf _{\rho \in K} \max _{x \in \bar{\Omega}} \frac{\left(1-\frac{1}{\alpha} F^{\prime}(\rho)\right) \Delta \rho+\lambda^{2} F(\rho)}{F^{\prime \prime}(\rho)|\nabla \rho|^{2}}
$$

where $K$ is the class of functions $\rho \in C^{(2, \delta)}(\bar{\Omega})$ such that

$$
\left.\rho\right|_{\partial \Omega}=u_{1}^{0}, \quad u_{1}^{0} \leq \rho(x)<u_{1}^{*}, x \in \bar{\Omega}
$$

is a necessary condition and the strict inequality is a sufficient condition of existence of solution $w(x) \in C^{(2, \delta)}(\bar{\Omega})$ of the problem (2.1), (2.2).

Proof. Let the strict inequality in (4.1) be satisfied. Then there exists a function $u_{0} \in K$ such that

$$
\Delta\left(u_{0}-\frac{1}{\alpha} F\left(u_{0}\right)\right)+\lambda^{2} F\left(u_{0}\right) \leq 0, \quad x \in \Omega,
$$




$$
u_{0}=u_{1}^{0}, \quad x \in \partial \Omega .
$$

From the upper solution $u_{0}(x)$ and lower solution $u(x) \equiv u_{1}^{0}$, we define two sequences of functions with the use of a monotone iterative scheme. Functions $U_{n}(x)$ are solutions of the problem

$$
\begin{gathered}
\Delta U_{n}-\alpha U_{n}=-\alpha u_{n-1}, \quad x \in \Omega, \\
U_{n}=0, \quad x \in \partial \Omega, \quad n=1,2, \ldots
\end{gathered}
$$

Functions $u_{n}(x)$ can be found from the equation

$$
U_{n}(x)=u_{n}(x)-\frac{1}{\alpha} F\left(u_{n}\right), \quad n=1,2, \ldots
$$

We show first that

$$
u_{1}(x) \leq u_{0}(x), \quad x \in \bar{\Omega} .
$$

Indeed, the function $v(x)=u_{0}(x)-\frac{1}{\alpha} F\left(u_{0}\right)-U_{1}(x)$ satisfies the problem

$$
\Delta v-\alpha v \leq 0, \quad x \in \Omega, \quad v=0, \quad x \in \partial \Omega .
$$

Then $v(x) \geq 0$ in $\Omega$ and

$$
U_{1}(x) \leq u_{0}(x)-\frac{1}{\alpha} F\left(u_{0}\right) .
$$

From this inequality and (4.4) for $n=1$ follows (4.5) since $u_{0}(x)<u_{1}^{*}$.

We show now by induction that

$$
u_{n}(x) \leq u_{n-1}(x), \quad x \in \bar{\Omega}, \quad n=1,2, \ldots
$$

If this inequality holds for $n=i$, then from (4.3), $U_{i+1}(x) \leq U_{i}(x), \quad x \in \bar{\Omega}$. Then from (4.4) follows (4.6) for $n=i+1$.

Since $u_{n}(x) \geq u_{1}^{0}$ in $\bar{\Omega}$, then there exists a limiting function

$$
\bar{u}(x)=\lim _{n \rightarrow \infty} u_{n}(x) .
$$

We show that it satisfies the problem (2.1),(2.2). Indeed, we have

$$
\begin{gathered}
\Delta\left(u_{n}-\frac{1}{\alpha} F\left(u_{n}\right)\right)+\lambda^{2} F\left(u_{n}\right)=\alpha\left(u_{n}-u_{n-1}\right), \quad x \in \Omega, \\
u_{n}=0, \quad x \in \partial \Omega .
\end{gathered}
$$

It remains to show that we can pass to the limit in (4.7). From convergence of the right-hand side in (4.3) in $L^{p}$ for any $p \geq 1$ follows convergence of $U_{n}$ in $W_{p}^{2}$ and by virtue of embedding theorems in $C^{(\delta)}$ for some positive $\delta$. Then from (4.4) follows convergence of $u_{n}$ in $C^{(\delta)}$. Again from (4.3) we obtain convergence of $U_{n}$ in $C^{(2, \delta)}$ and from (4.4) convergence of $u_{n}$ in $C^{(2, \delta)}$. Thus the limiting function $\bar{u}(x)$ satisfies the limiting problem.

To prove the necessity we note that if a solution $w(x) \in C^{(2, \delta)}$ of the problem $(2.1),(2.2)$ exists, then it belongs to $K$ and it satisfies equality in (4.1). The theorem is proved.

Remark 4.2. We note that the necessary and the sufficient conditions do not coincide exactly. The equality in (4.1) does not guarantee existence of a classical solution if infimum of the functional is reached on the critical solution. 
In the end of this section we present an example illustrating application of the minimax representation to estimate the critical size of the domain.

We consider the problem (1.3), (1.4) in the case of one spatial variable $0 \leq x \leq L$ (here $\lambda=L$ ), and assume that $F\left(u_{1}\right)=\exp \left(z u_{1}\right)(c f .[2,6])$. Here $z$ is a positive parameter called the Zeldovich number. Nonlinearities of this form are specific for combustion processes. In this case the problem can be solved explicitly and we can compare exact and approximate critical conditions. Now by the way of the minimax conditions, we can use test-functions to approximate $L$. We put as test function

$$
\rho(x)=a x^{2}+b x+u_{1}^{0},
$$

where

$$
a=-\frac{e^{z\left(u_{m}+u_{1}^{0}\right)}}{2\left(1-\frac{z}{\alpha} e^{z\left(u_{m}+u_{1}^{0}\right)}\right)}, \quad b=2 \sqrt{-a u_{m}}, \quad L=2 \sqrt{u_{m} /-a},
$$

$u_{m}$ is a parameter. Then

$$
\rho(L / 2)=u_{m}+u_{1}^{0} .
$$

We impose an additional condition

$$
\rho^{\prime}(L / 2)=0
$$

In this case the maximum of the function $\rho(x)$ is reached at $L / 2$ and equals $s=u_{m}+u_{1}^{0}$. We require that

$$
1-\frac{z}{\alpha} e^{z s}>0
$$

Then $a$ is negative and all expressions above are well defined. We note that at $s=u_{1}^{*}$ the last inequality becomes an equality, so that $u_{m}+u_{1}^{0}<u_{1}^{*}$.

It can be verified directly that

$$
\left(1-\frac{1}{\alpha} F^{\prime}(\rho)\right) \rho^{\prime \prime}+F(\rho)<0
$$

and according to the minimax condition, there exists a solution of the problem $(2.1),(2.2)$.

From (4.8) we find

$$
L=2 \sqrt{2\left(s-u_{1}^{0}\right) e^{-z s}\left(1-e^{z} \alpha e^{z s}\right)} .
$$

The function $L_{a p}(s)$ defined by formula (4.9) is positive for $u_{1}^{0}<s<u_{1}^{*}$. It gives an approximation of the length of the interval $L$ for each value of the maximal temperature $s$. It has a maximum at some $s=s^{*}$, it is increasing at the interval $u_{1}^{0}<s<s^{*}$ and decreasing at the interval $s^{*}<s<u_{1}^{*}$.

For any given $L_{0}, 0<L_{0}<L_{a p}\left(s^{*}\right)$ there exist two solutions, $s_{0}$ and $s_{1}, s_{0}<s_{1}$ of the equation $L_{a p}(s)=L_{0}$. To each of them corresponds an upper function of the problem (2.1), (2.2). Then for $L=L_{0}$ there exists an exact solution of this problem with the maximal value of its first component less than $s_{0}$. Hence we can conclude that for the function $L_{e x}(s)$ which corresponds to the exact solutions,

$$
L_{a p}(s)<L_{e x}(s), \quad u_{1}^{0}<s<s^{*}
$$

and

(see Fig. 3).

$$
L_{a p}\left(s^{*}\right)<\max _{s} L_{e x}(s)
$$

Eventually, Figure 4 shows the maximal length of the interval as a function of $\frac{1}{\alpha}$. 


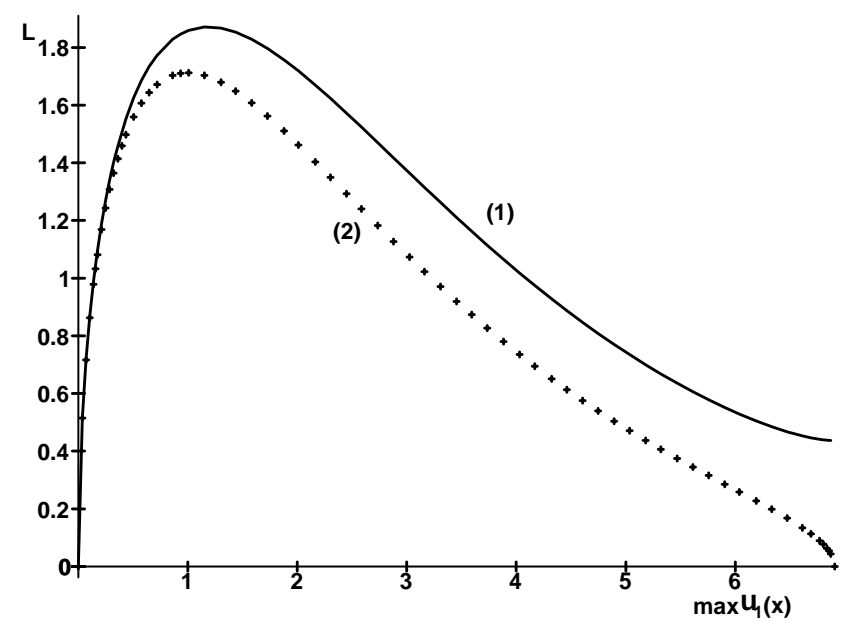

FIGURE 3. Length of the interval as a function of maximal particles temperature $\theta_{1}$ for stationary solutions with $F(u)=e^{u}$ and $\alpha=1000$. (1) exact solution, (2) approximate solution.

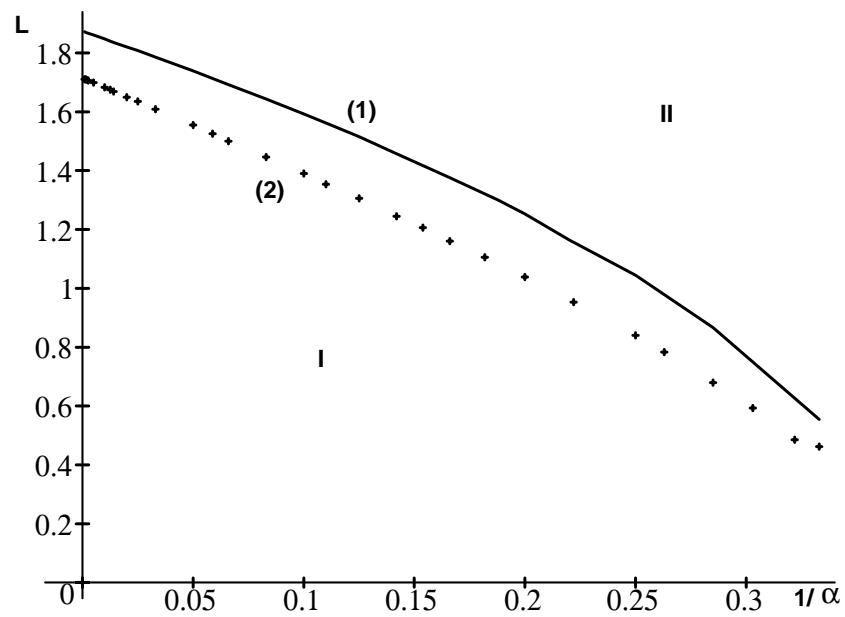

FIgure 4. Critical condition of heat explosion: maximal length of the interval as a function of $\frac{1}{\alpha}$ where a stationary solution exists. (1) Exact solution. (2) Estimation. Corresponding domains: I Stationary solutions exist, II Stationary solutions do not exist. 
We note that a wider class of test functions $\rho$ can be considered. In particular it can be functions from $C^{(\delta)}$ if we assume that there exists a finite number of points of discontinuity of the first derivative and at each point $x_{0}, \rho^{\prime}\left(x_{0}-0\right) \geq \rho^{\prime}\left(x_{0}+0\right)$.

We can use also a piece-wise linear function as a test function. It coincides with the exact critical solution where the maximum of the first component equals $u_{1}^{*}$. The corresponding length of the interval is

$$
L^{*}=\frac{2}{\sqrt{\alpha}} \ln \frac{\alpha}{z} .
$$

\section{Conclusions}

In this work we study a model which describes heat explosion in a non-moving heterogeneous medium consisting of reacting particles surrounded by a gas. Chemical reaction can occur inside the particles or between the particles and the gas, but it does not occur inside the gaseous phase. The particles are sufficiently small and there is no heat conduction in this phase. The gas is heat conducting and there is heat exchange between the two phases. The heterogeneous medium heated due to exothermic chemical reaction loses its energy by heat conduction between the gas and the vessel's cold boundaries which are maintained at the initial (room) temperature.

We consider a two-temperature continuous model and study existence and properties of solutions. We show that there are two critical sizes of the domain. One of them corresponds to loss of regularity of solutions, another one to disappearance of solutions when two solutions merge. From the physical point of view the first case corresponds to local heat explosion on one particle, the second case to global heat explosion where the solution of the initial boundary value problem becomes unbounded (blow-up solution). We obtain a critical condition of existence of solutions in a minimax form. It allows to estimate explicitly the critical size of the domain. For the Arrhenius type kinetic with $F\left(u_{1}\right)=\exp \left(u_{1}\right)$, the critical condition obtained above performed for one dimensional domains coincides with previous results [2].

One of the open questions here is which of these two critical sizes corresponds to larger domains. Another way to put this question is what type of heat explosion occurs when we increase the domain. Our conjecture is that global heat explosion occurs in larger domains than the local one. It is also connected with stability of solutions. We see on an example that the critical solution lies on decreasing branches and, consequently, can be expected to be unstable.

We show that solutions of the two-phase problem converge to solutions of a problem in a homogeneous medium when the coefficient of heat exchange between two phases increases.

On the other hand heat explosion in particles-gas system is connected with Semenov's theory of heat explosion (see [14]). If $u_{1}$ is the temperature of a reacting medium and $u_{2}$ is an ambient temperature, then according to this theory heat explosion occurs if the equation

$$
F\left(u_{1}\right)-\alpha\left(u_{1}-u_{2}\right)=0
$$

does not have solutions. In the problem of heat explosion of the two-phase medium, $u_{1}$ corresponds to the temperature of the particles, $u_{2}$ to the temperature of the surrounding gas. We suppose that this equation is solvable for $u_{2}=0$, i.e. the particles do not explode on the boundary of the domain. A stationary temperature distribution with a bounded temperature inside the domain can exist if there are no local heat explosion or if it occurs on (m-1)-dimensional surfaces (but not in m-dimensional domains). However, in the latter case, this distribution is probably unstable and a small temperature distribution will lead to extinction or to global heat explosion. 


\section{REFERENCES}

[1] E.V. Chernenko and V.I. Rozenband, Calculation of the extremal combustion characteristics of aerial suspensions of metal with autoignition. Combustion, Explosion and Shock Waves 16 (1980) 3-10.

[2] I.G. Dik and A.Yu. Krainov, Ignition regims of a gas suspension in a vessel with heated walls. Combustion, Explosion, and Shock Waves 20 (1984) 58-61.

[3] W.H. Fleming and R. Rishel, An integral formula for total gradient variation. Arch. Math. 11 (1960) $218-222$.

[4] T. Gallouet, F. Mignot and J.-P. Puel, Quelques résultats sur le problème $-\Delta u=\lambda e^{u}$. C. R. Acad. Sci. Paris Sér. I 307 (1988) 289-292.

[5] Y. Giga, R. Kohn, Nondegeneracy of blowup for semilinear heat equations. Comm. Pure Appl. Math. XLII (1989) 845-884.

[6] M.A. Gurevich, G.E. Ozerova and A.M. Stepanov, Ignition limit of a monofractional gas suspension. Combustion, Explosion, and Shock Waves 10 (1974) 88-93.

[7] N.V. Krylov, Lectures on elliptic and parabolic equations in Hölder spaces, AMS, Graduate studies in Mathematics (1996).

[8] V.I. Lisitsyn, E.N. Rumanov and B.I. Khaikin, Induction period in the ignition of a particle system. Combustion Explosion, and Shock Waves 1 (1971) 1-6.

[9] N. Mizogushi and T. Suzuki, Equations of gas combustion: S-shaped bifurcations and mushrooms. J. Differential Equations 134 (1997) 183-205.

[10] R.E. O'Malley and L.V. Kalachev, Regularization of nonlinear differential-algebraic equations. SIAM J. Math. Anal. 25 (1994) 615-629.

[11] E.N. Rumanov and B.I. Khaikin, Critical autoignition conditions for a system of particles. Combustion, Explosion, and Shock Waves 5 (1969) 129-136.

[12] A.I. Volpert, The spaces BV and quasilinear equations. Math USSR - Sbornik 2 (1967) 225-267.

[13] A.I. Volpert, S. Hudjaev, Analysis in classes of discontinuous functions and equations of mathematical physics, Martinus Nijhoff Publishers, Dordrecht (1985).

[14] Ya. B. Zeldovich, G.I. Barenblatt, V.B. Librovich and G.M. Makhviladze, The mathematical theory of combustion and explosion. Plenum Press, New York-London (1985). 